\title{
Intracystic papillary carcinoma of breast: interrelationship with in situ and invasive carcinoma and a proposal of pathogenesis: array comparative genomic hybridization study of 14 cases
}

\author{
Thaer Khoury ${ }^{1}$, Qiang $\mathrm{Hu}^{2}$, Song $\mathrm{Liu}^{2}$ and Jianmin Wang ${ }^{2}$ \\ ${ }^{1}$ Department of Pathology, Roswell Park Cancer Institute, Buffalo, NY, USA and ${ }^{2}$ Department of Biostatistics, \\ Roswell Park Cancer Institute, Buffalo, NY, USA
}

\begin{abstract}
Classifying intracystic papillary carcinoma under invasive or in situ ductal carcinoma is still a matter of debate. The purpose of this study was to explore the genomic relationship of this tumor to its concurrent invasive ductal carcinoma and ductal carcinoma in situ using array comparative genomic hybridization. Intracystic papillary carcinoma cases were classified into three categories: pure, with concurrent ductal carcinoma in situ or with concurrent invasive ductal carcinoma. Each component was dissected using laser capture microdissection. DNA was extracted and array comparative genomic hybridization was performed. The test of difference in copy number changes among the three tumors was carried out using CGHMultiArray. Intracystic papillary carcinoma clustered with four of five concurrent ductal carcinoma in situ cases and with two of two invasive ductal carcinoma cases. Intracystic papillary carcinoma showed the highest proportions of genome copy number aberration, followed by ductal carcinoma in situ, and then by invasive ductal carcinoma $(P=0.06)$. Comparing intracystic papillary carcinoma with invasive ductal carcinoma vs without invasive ductal carcinoma, the former had 11q22.1-23.3 loss $(P=0.031)$ and chr5 gain $(P=0.085)$, and was enriched with matrix metalloproteinase genes. Comparing intracystic papillary carcinoma with ductal carcinoma in situ vs without ductal carcinoma in situ, the former had gain in 5q35.3 $(P=0.041), 8 q 24.3(P=0.041)$ and $21 q 13.2$ to 21q13.31 $(P=0.011)$. Comparing intracystic papillary carcinoma with ductal carcinoma in situ, the latter acquired a group of genes involved in cell adhesion and motility, whereas intracystic papillary carcinoma differentially expressed genes that are involved in papillary carcinomas of other organs (thyroid and kidney). We conclude that the overall molecular change in intracystic papillary carcinoma is closer to ductal carcinoma in situ than to invasive ductal carcinoma, which may explain the indolent behavior of this tumor. We offer herein a proposal of intracystic papillary carcinoma pathogenesis through its relation to invasive ductal carcinoma and ductal carcinoma in situ.

Modern Pathology (2014) 27, 194-203; doi:10.1038/modpathol.2013.136; published online 2 August 2013
\end{abstract}

Keywords: array comparative genomic hybridization; intracystic papillary carcinoma; pathogenesis

Intracystic papillary carcinoma is a distinctive variant of papillary ductal carcinoma, confined to a dilated cystic space and surrounded by a fibrous

Correspondence: Dr T Khoury, MD, Department of Pathology, Roswell Park Cancer Institute, Elm \& Carlton Streets, Buffalo, NY 14263, USA or Dr J Wang, PhD, Department of Biostatistics, Roswell Park Cancer Institute, Elm \& Carlton Streets, Buffalo, NY 14263, USA.

E-mail: jianmin.wang@roswellpark.org or

thaer.khoury@roswellpark.org

Received 27 March 2013; revised 3 June 2013; accepted 4 June 2013; published online 2 August 2013 capsule, and characterized by thin fibrovascular stalks devoid of a myoepithelial cell layer, and a neoplastic cell population with histological features characteristic of low-grade ductal carcinoma in situ. It can be present as an isolated lesion or associated with conventional non-papillary ductal carcinoma in situ and/or invasive ductal carcinoma. When it occurs as an isolated lesion with no concurrent ductal carcinoma in situ or invasive ductal carcinoma, the tumor has favorable prognosis with no reported lymph node metastases or disease-related death. The presence of ductal carcinoma in situ or 
invasive ductal carcinoma in the surrounding breast tissue is associated with increased risk of local recurrence for the former and local and metastatic rates in the latter. ${ }^{1}$

The classification of intracystic papillary carcinoma as a form of invasive ductal carcinoma or ductal carcinoma in situ is controversial. Although the absence of myoepithelial cell layer would suggest intracystic papillary carcinoma as a form of invasive ductal carcinoma, the presence of collagen IV would suggest that it is a form of ductal carcinoma in situ. ${ }^{2-5}$ The WHO Working Group reached a consensus that intracystic papillary carcinoma should be staged and managed like ductal carcinoma in situ. ${ }^{1}$

Genetic alterations in the form of interstitial deletions, loss of heterozygosity at $16 q$ and $1 p$, and numerical and structural alterations at chromosomes $16 q$ and $1 p$ with fusion of chromosome 16 and 1 (der(1;16)) have been described in intracystic papillary carcinoma. ${ }^{6-8}$ Duprez et $a l^{9}$ studied the genomic characterization of papillary carcinoma including intracystic type and compared it with non-concurrent invasive ductal carcinoma. In addition, many studies have attempted to classify intracystic papillary carcinoma using immunohistochemistry. ${ }^{2-5}$

This is the first study that compared between intracystic papillary carcinoma with concurrent ductal carcinoma in situ and invasive ductal carcinoma on the genomic level. We propose an evolutionary pathway between these three entities.

\section{Materials and methods}

\section{Cases}

Clinical and pathologic databases were searched for 'intracystic', 'encapsulated' or 'papillary' carcinoma of the breast at Roswell Park Cancer Institute between 1992 and 2010. Only cases that had available slides and tissue blocks are included in the study.

The original slides of these cases were reviewed. The pathologic features including tumor size (invasive ductal carcinoma and intracystic papillary carcinoma), lymph node status, nuclear grade and margin status were recorded. Myoepithelial markers including p63 (clone 4A4 (Dako), with 1:50 dilution, and EDTA antigen retrieval), smooth muscle myosin (clone SMMS-1 (Cell Marque), prediluted and EDTA antigen retrieval) and Calponin (clone CALP (Dako), with dilution 1:200, and Vector antigen retrieval) were performed on all cases. Complete or partial loss of myoepithelial cell layer was required for the diagnosis of intracystic papillary carcinoma. ${ }^{1}$ We adopted the WHO definition of invasion in intracystic papillary carcinoma as a tumor that assumes a pattern of invasive ductal carcinoma and lacks the papillary architecture. ${ }^{1}$ Invasive ductal carcinoma was graded using modified Bloom Richardson grading system. ${ }^{10}$ For a lesion to qualify to be ductal carcinoma in situ, the involved ducts had to be completely surrounded by the myoepithelial cell layer with non-papillary growth pattern. Ductal carcinoma in situ was graded based on European Pathologists Working Group classification system. ${ }^{11}$ All cases were stained with estrogen receptor (clone 1D5 (Dako), with 1:100 dilution, and TRS/Vector antigen retrieval) and progesterone receptor (PgR636 (Dako), with dilution 1:100, and TRS/Steamer antigen retrieval).

\section{Laser Capture Microdissection}

Paraffin-embedded breast tissue was cut at $8 \mu \mathrm{m}$ and mounted on polyethylene naphthalate-membranecoated slides (Leica, Wetzlar, Germany; cat. no. 11505158) and dried overnight at room temperature. The next day slides were deparaffinized in three changes of xylene, rehydrated using graded alcohols and stained manually with hematoxylin and eosin. All the solutions were prepared with doubledistilled water and changed for the each sample. The laser capture microdissection was carried out using Leica Laser Microdissection systems. DNA was extracted from the laser capture microdissection tissues by QIAamp DNA Micro Kit (Qiagen; cat. no. 56304 ) and eluted in $20 \mu \mathrm{l}$ nuclease-free water, and the concentration of DNA was measured by NanoDrop, Spectrophotometer, ND-1000.

\section{Array Comparative Genomic Hybridization Analysis}

One microgram of laser capture microdissection captured formalin-fixed, paraffin-embedded tumor genomic DNA was Cy5 labeled (test) and $1 \mu \mathrm{g}$ of pooled control DNA from 20 healthy male donors was Cy3 labeled (reference) using the Bioarray Kit (Enzo Life Sciences). Following purification, equal molar aliquots of the test and reference probes were combined, denatured and hybridized to an RPCI 21K BAC array (Roswell Park Cancer Institute). ${ }^{12}$ After a 20-h hybridization, the arrayed slides were washed and then scanned on a GenePix 4200AL Scanner (Molecular Devices) to generate highresolution ( $5 \mu \mathrm{m})$ images for both Cy5 (test) and Cy3 (reference) channels. Image analysis was performed using ImaGene (version 8.0.0) software from BioDiscovery. The $\log 2$ test/reference ratios were normalized using a subgrid loess correction. Mapping information was added to the resulting $\log 2$ test/ reference values. The mapping data for each BAC was found by querying the human genome sequence at http://genome.ucsc.edu.

\section{Statistical Analysis}

A loess-corrected $\log 2$ ratio of the background subtracted test/control was calculated for each clone. The data are further normalized using median-based 
normalization and segmented using DNAcopy. ${ }^{13}$ Instead of using a fixed cutoff value, copy number gain/loss was called by CGHcall with $75 \%$ assumed tumor purity. ${ }^{14}$ The test of difference in copy number changes among intracystic papillary carcinoma, ductal carcinoma in situ and invasive ductal carcinoma was carried out using CGHMultiArray. ${ }^{15}$ We performed two analyses for the genomic variation in intracystic papillary carcinoma cases, one based on the presence or absence of concurrent ductal carcinoma in situ (intracystic papillary carcinoma with ductal carcinoma in situ $(n=6)$ vs intracystic papillary carcinoma without ductal carcinoma in situ $(n=8)$ ) and one based on the presence or absence of concurrent invasive ductal carcinoma (intracystic papillary carcinoma with invasive ductal carcinoma $(n=6)$ vs intracystic papillary carcinoma without invasive ductal carcinoma $(n=8))$. Only three cases had concurrent ductal carcinoma in situ and invasive ductal carcinoma, precluding statistical analysis. Genomic variation analysis in intracystic papillary carcinoma group with relation to patient age, tumor grade and tumor recurrence was also performed. Finally, we compared the genomic variation between intracystic papillary carcinoma and concurrent ductal carcinoma in situ and invasive ductal carcinoma. An Online Mendelian Inheritance in Man (OMIM) search was performed on all the involved genes in the genomic variation.

\section{Results}

\section{Cases}

A total of 22 intracystic papillary carcinoma cases were identified in the pathology database at Roswell Park Cancer Institute between 1992 and 2010. Twelve cases had concurrent ductal carcinoma in situ, whereas nine cases had concurrent invasive ductal carcinoma. Concurrent intracystic papillary carcinoma, ductal carcinoma in situ and invasive ductal carcinoma were identified in four cases (Figure 1). Array comparative genomic hybridization test was successful in 14 intracystic papillary carcinoma (5 grade I and 9 grade II), 6 ductal carcinoma in situ (4 grade I and 2 grade II with comedo-type necrosis) and 4 invasive ductal carcinoma (all modified Scarff Bloom Richardson grade I). The median size and range of intracystic papillary carcinoma was $17 \mathrm{~mm}$ (6-47 mm). All invasive tumors were of invasive ductal carcinoma type with the median and range of tumor size $5 \mathrm{~mm}(1-7 \mathrm{~mm})$. Although five cases had array comparative genomic hybridization results on intracystic papillary carcinoma with concurrent ductal carcinoma in situ, these results were available for intracystic papillary carcinoma with concurrent invasive ductal carcinoma in two cases. Metastatic carcinoma to sentinel lymph node was seen in 1 of 13 cases. All cases had
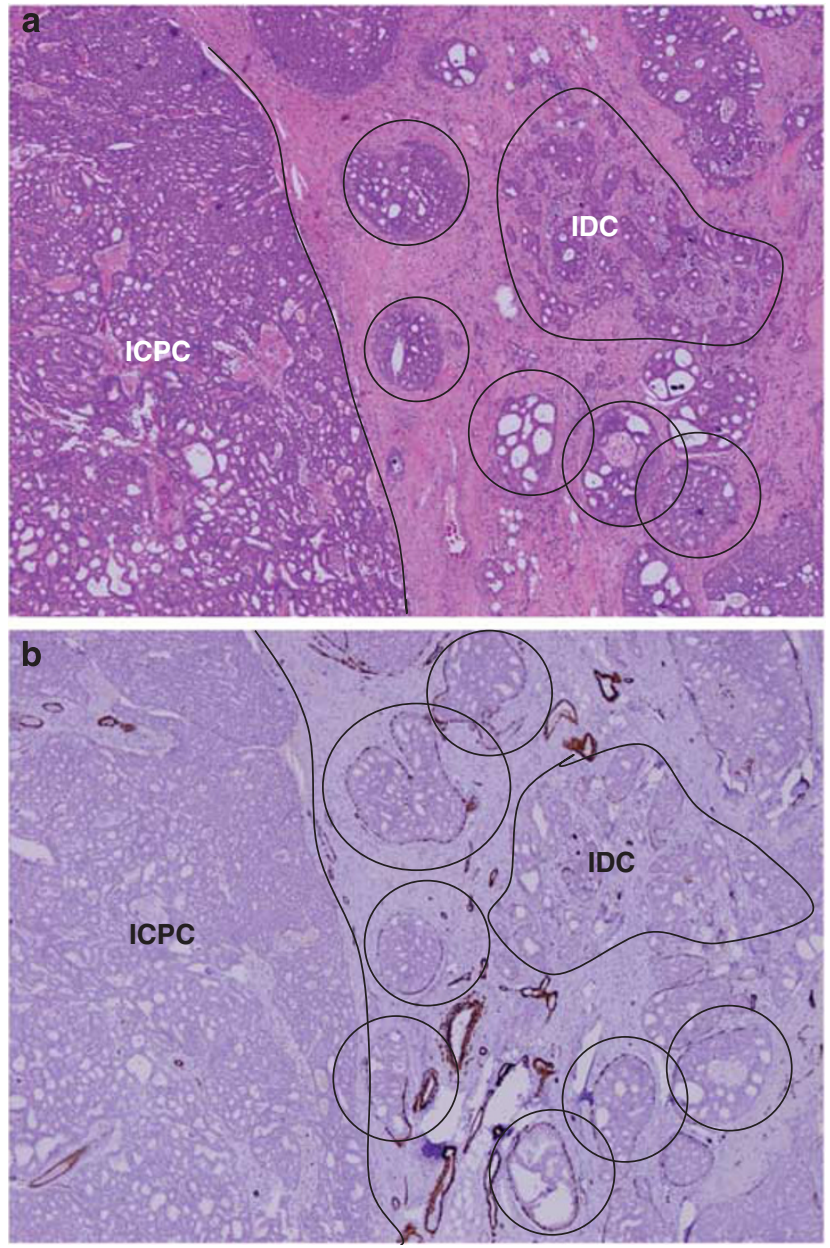

Figure 1 Hematoxylin and eosin (a) and smooth muscle myosin (SMM) (b) immunostain figures of intracystic papillary carcinoma, invasive ductal carcinoma and ductal carcinoma in situ (in circles) $(\times 4)$.

negative surgical resection margins, with one case having close margin $(1 \mathrm{~mm})$. All cases were estrogen receptor positive and progesterone receptor positive. Four $(18 \%)$ cases had nuclear grade I and $18(82 \%)$ had nuclear grade II.

All patients were women except for one (patient no. 1). All women were postmenopausal except for two who were perimenopausal. The patients' age ranged from 51 to 94 years with a median of 73 years. All tumors were surgically removed (excision or mastectomy). Therapy modality was available for all patients except for four. One patient had chemotherapy. However, this case was excluded from the analysis because of the unsuccessful array comparative genomic hybridization. The time of follow-up range was 1-117 months with a median of 36.5 months. Two patients were lost to follow-up. Three patients developed local recurrence, two contralateral in the form of invasive ductal carcinoma and one ipsilateral in the form of papillary carcinoma. All patients who developed local recurrence had concurrent ductal carcinoma in situ (Table 1). 
Table 1 Clinicopathological findings of studied cases

\begin{tabular}{|c|c|c|c|c|c|c|c|c|c|c|}
\hline Case no. (grade) & DCIS (grade) & $I D C$ & $\begin{array}{c}I D C \text { —size } \\
(\mathrm{mm})\end{array}$ & $\begin{array}{l}\text { ICPC_size } \\
(\mathrm{mm})\end{array}$ & Age (years) & Recurrence & $\begin{array}{l}\text { Site of } \\
\text { recurrence }\end{array}$ & $\begin{array}{c}\text { Follow-up } \\
\text { time (months) }\end{array}$ & $\begin{array}{l}\text { Adjuvant } \\
\text { therapy }\end{array}$ & $\begin{array}{l}\text { Type of } \\
\text { surgery }\end{array}$ \\
\hline ICPC1 (II) & No & No & 0 & 21 & 76 & & & NA & NA & NA \\
\hline ICPC2 $^{\mathrm{a}}$ (II) & Yes (II) & No & 0 & 13 & 70 & & & 26 & NA & Excision \\
\hline ICPC3 ${ }^{\mathrm{a}}$ (II) & $\operatorname{Yes}^{\mathrm{a}}(\mathrm{I})$ & No & 0 & 10 & 70 & Yes & Contralateral & 117 & NA & Mastectomy \\
\hline $\mathrm{ICPC}^{\mathrm{a}}(\mathrm{I})$ & Yes (I) & Yes & 1 & 30 & 80 & & & NA & NA & Excision \\
\hline ICPC5 $^{\mathrm{a}}$ (I) & Yes (II) & No & 0 & 20 & 79 & & & 37 & Radiation & Excision \\
\hline ICPC6 (II) & No & No & 0 & 10 & 78 & & & 30 & Chemo & Excision \\
\hline ICPC7 (II) & No & Yes $^{\mathrm{a}}$ & 1 & 10 & 94 & & & 49 & Hormonal & Mastectomy \\
\hline ICPC8 $^{a}$ (II) & Yes (I) & No & 0 & 20 & 53 & & & 22 & Radiation & Excision \\
\hline ICPC9 (II) & Yes (I) & No & 0 & 15 & 82 & & & 60 & $\begin{array}{l}\text { Radiation and } \\
\text { hormonal }\end{array}$ & Excision \\
\hline ICPC10a (II) & No & No & 0 & 9 & 55 & & & 55 & Hormonal & Mastectomy \\
\hline ICPC11 (II) & Yes $^{\mathrm{a}}(\mathrm{I})$ & No & 0 & 6 & 76 & Yes & Contralateral & 58 & Surgery only & Mastectomy \\
\hline ICPC12a (II) & Yes $^{\mathrm{a}}$ (II) & Yes & 6 & 45 & 61 & & & 39 & Hormonal & Excision \\
\hline $\mathrm{ICPC} 3^{\mathrm{a}}$ (I) & Yes $^{\mathrm{a}}(\mathrm{I})$ & No & 0 & 35 & 64 & Yes & Ipsilateral & 19 & Surgery only & Excision \\
\hline ICPC14 (II) & No & Yes & 5 & 15 & 89 & & & 47 & Hormonal & Excision \\
\hline ICPC15ª (II) & $\mathrm{Yes}^{\mathrm{a}}$ (II) & No & 0 & 12 & 70 & & & 38 & Hormonal & Excision \\
\hline ICPC16 $^{\text {a }}$ (II) & $\mathrm{Yes}^{\mathrm{a}}$ (II) & Yes & 6 & 47 & 64 & & & 35 & $\begin{array}{l}\text { Radiation and } \\
\text { hormonal }\end{array}$ & Excision \\
\hline ICPC17 (II) & Yes (II) & No & 0 & 12 & 51 & & & 29 & Hormonal & Mastectomy \\
\hline $\mathrm{ICPC}^{\mathrm{a}}{ }^{\mathrm{a}}$ (I) & No & Yes $^{a}$ & 7 & 9 & 61 & & & 20 & Hormonal & Excision \\
\hline ICPC19a (I) & Yes (I) & Yes & 5 & 9 & 68 & & & 12 & $\begin{array}{l}\text { Radiation and } \\
\text { hormonal }\end{array}$ & Excision \\
\hline ICPC20 $0^{\mathrm{a}}$ (II) & No & $\mathrm{Yes}^{\mathrm{a}}$ & 5 & 24 & 79 & & & 1 & Radiation & Excision \\
\hline ICPC21 ${ }^{\mathrm{a}}$ (II) & No & No & 0 & 40 & 78 & & & 46 & $\begin{array}{l}\text { Radiation and } \\
\text { hormonal }\end{array}$ & Excision \\
\hline ICPC22 (II) & No & $\mathrm{Yes}^{\mathrm{a}}$ & 5 & 19 & 81 & & & 36 & Hormonal & Excision \\
\hline $\begin{array}{l}\text { Total Median } \\
\text { (range) }\end{array}$ & 12 & 9 & $5(1-7)$ & $17(6-47)$ & 73 & 3 & & $36.5(1-117)$ & & \\
\hline
\end{tabular}

Abbreviations: aCGH, array comparative genomic hybridization; ICPC, intracystic papillary carcinoma; DCIS, ductal carcinoma in situ; IDC, invasive ductal carcinoma; NA, data not available; al IDC cases were mSBR grade I. 


\section{Array Comparative Genomic Hybridization Findings}

Genome profile and variation of intracystic papillary carcinoma. Intracystic papillary carcinoma showed $16 \mathrm{p}$ gain, $16 \mathrm{q}$ loss and $1 \mathrm{q}$ gain. In addition, it revealed some novel large-scale copy number variations, $7 q$ loss (4 of 14 cases), 8p23.3-21.1 loss (5 of 14 cases), 11q14.1-22.1 loss (4 of 14 cases), 11q22.1-25 loss (5 of 14 cases) and 9p13.1-12 loss (5 of 14 cases) (Figure 2).

The genomic variation between intracystic papillary carcinoma with ductal carcinoma in situ $(n=6)$ $v s$ without ductal carcinoma in situ $(n=8)$ showed that intracystic papillary carcinoma without ductal carcinoma in situ had 5q35.3 gain (from 176474585 to 180175485 , in 3 of 4 cases) vs 1 of 10 intracystic papillary carcinoma with ductal carcinoma in situ $(P=0.041)$. Intracystic papillary carcinoma without ductal carcinoma in situ had 8q24.3 gain (from 142015488 to 145957473 , in 3 of 4 cases) vs 1 of 10 intracystic papillary carcinoma with ductal carcinoma in situ $(P=0.041)$. Finally, intracystic papillary carcinoma without ductal carcinoma in situ had 21q13.2-13.31 gain (from 42127232 to 44695209 in 3 of 4 cases) VS 0 of 10 intracystic papillary carcinoma with ductal carcinoma in situ $(P=0.011)$ (Figure 3). The genes involved are listed in Supplementary Table 1.

The genomic variation between intracystic papillary carcinoma with invasive ductal carcinoma $(n=6)$ vs without invasive ductal carcinoma $(n=8)$ showed that the latter had 11q22.1-23.3 loss in 6 of 8 cases vs 0 of 6 in the former $(P=0.031)$. Similarly, chr5 gain was seen in four of eight cases (an additional case also had gain of part of chr5) in intracystic papillary carcinoma without invasive ductal carcinoma vs 0 of 6 in intracystic papillary carcinoma with invasive ductal carcinoma with borderline significance $(P=0.085)$ (Figure 4). The involved genes are listed in Supplementary Table 2. The following genes are of interest, matrix metalloproteinases (MMP) 1, 3, 7, 8, 10, 12, 13 and 27.

There was no genomic variation with relation to the nuclear grade, patient age or the risk for local recurrence (Figure 5).

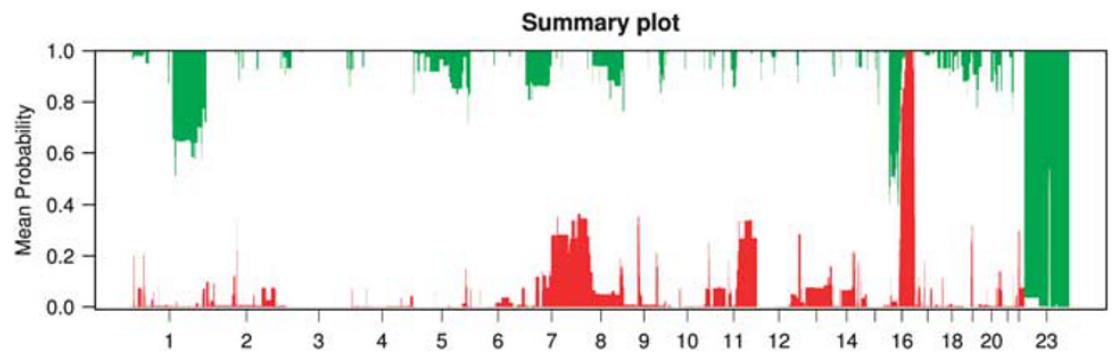

Figure 2 Frequency plot of copy number gains and losses in intracystic papillary carcinoma cases. The proportion of tumors in which each bacterial artificial chromosome (BAC) clone is gained (green bars) or lost (red bars) is plotted (y axis) for each BAC clone according to its genomic position ( $x$ axis).

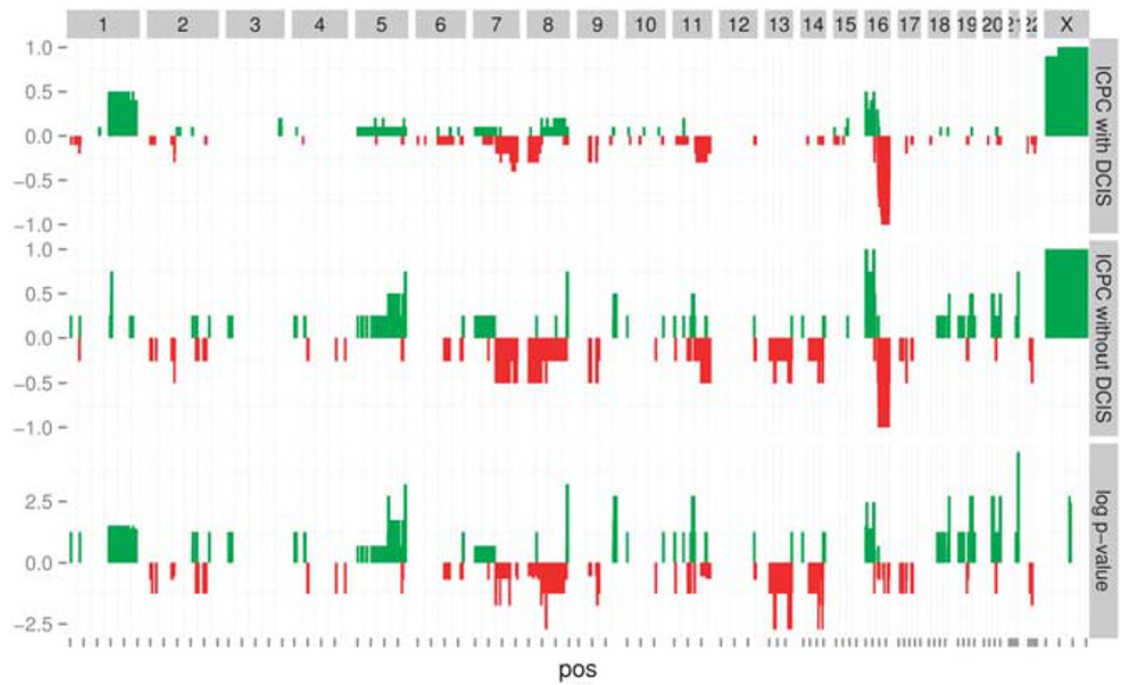

Figure 3 Frequency plot of copy number gains and losses in intracystic papillary carcinoma (ICPC) with ductal carcinoma in situ (DCIS) comparing with ICPC without DCIS. Significant changes included 5q35.3 gain, 8q24.3 gain and 21q13.2-13.31 gain in ICPC without DCIS. 


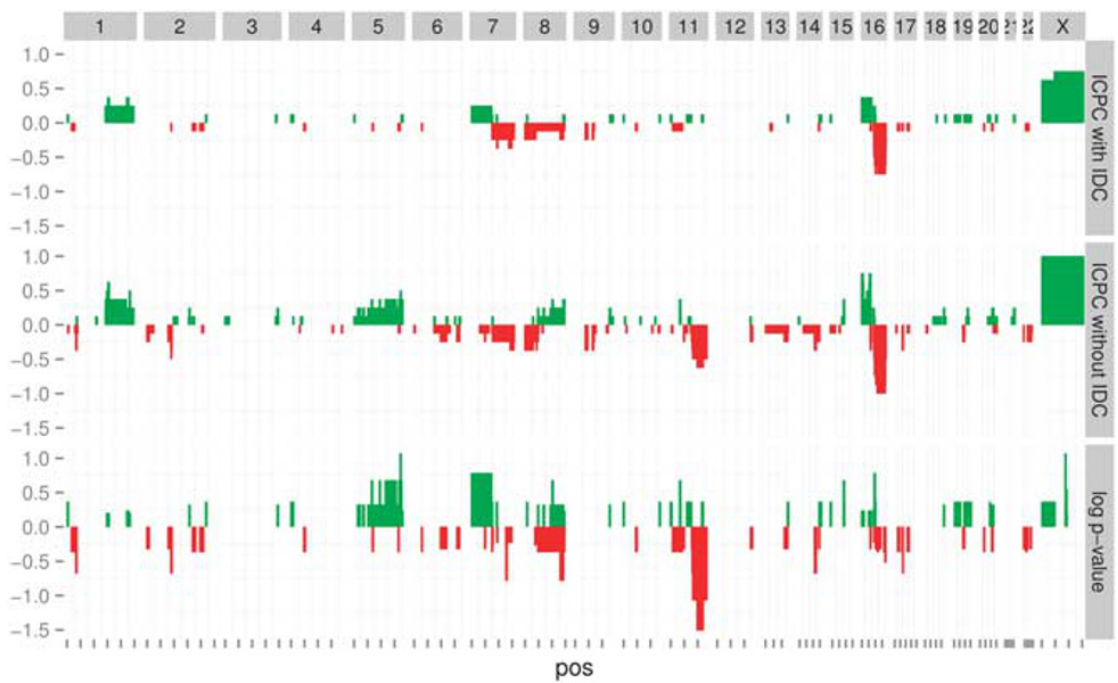

Figure 4 Frequency plot of copy number gains and losses in intracystic papillary carcinoma (ICPC) with invasive ductal carcinoma (IDC) comparing with ICPC without IDC. Significant changes included chr5 gain and 11q22.1-23.3 loss in ICPC without IDC.

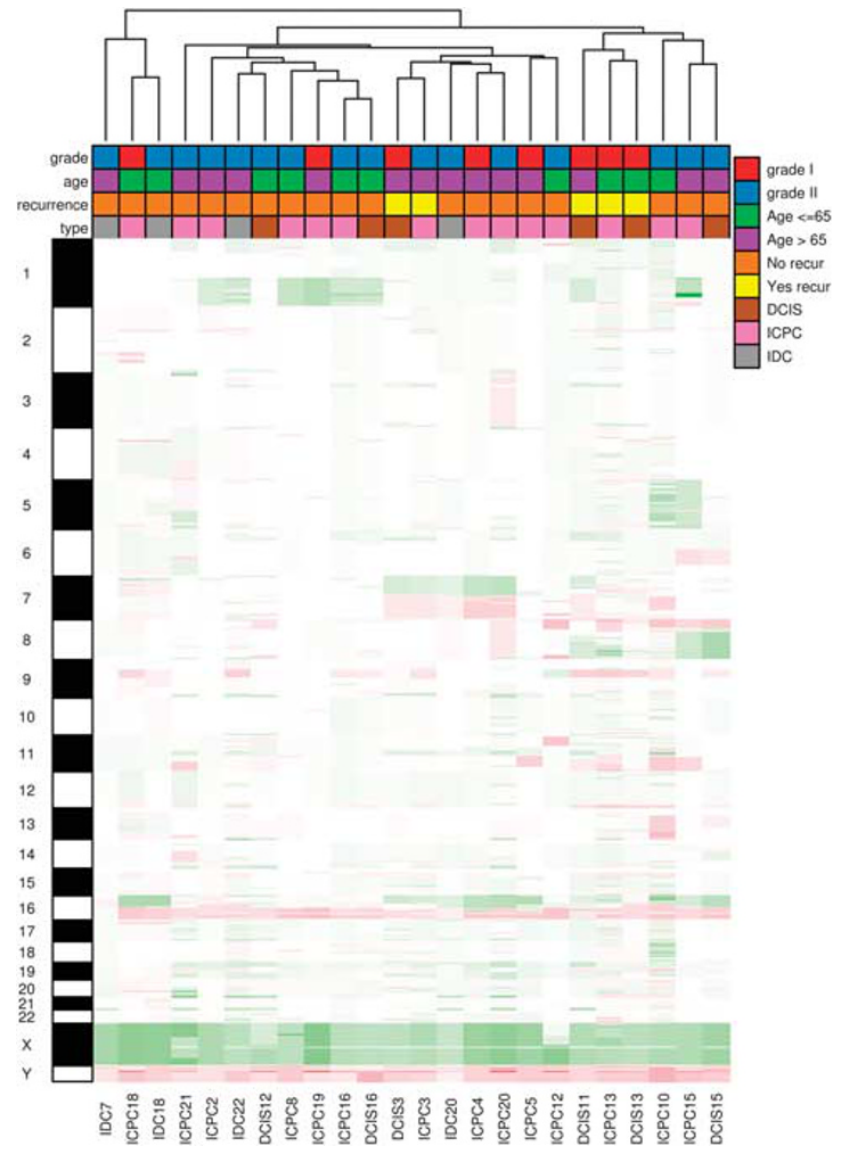

Figure 5 Unsupervised hierarchical clustering analysis of intracystic papillary carcinoma (ICPC) with concurrent invasive ductal carcinoma (IDC) and ductal carcinoma in situ (DCIS). Hierarchical cluster analysis performed with array comparative genomic hybridization categorical states (ie gains, losses) using the Euclidean distance metric and the Wards algorithm. Note the clustering of ICPC with the concurrent DCIS or IDC. No clustering was noted in relation to tumor recurrence, grade or age.
Overall genomic variation between intracystic papillary carcinoma, ductal carcinoma in situ and invasive ductal carcinoma. Hierarchical agglomerative clustering of samples using probe $\log 2$ ratio shows that the three types could not be separated into different clusters. Four of five concurrent intracystic papillary carcinomas with ductal carcinoma in situ clustered together. Both invasive ductal carcinomas clustered with the concurrent intracystic papillary carcinoma (Figures 5 and 6). Pearson's correlation between foci of the sample patient using $\log 2$ ratio ranges from 0.49 to 0.84 with a median value of 0.73 .

The total genome copy number change (gain or loss) for intracystic papillary carcinoma $(n=14)$, ductal carcinoma in situ $(n=6)$ and invasive ductal carcinoma $(n=4)$ was 315 segments, 100 segments and 60 segments, respectively, whereas the median and range was 14.5 (4-68), 13.5 (11-29) and 5 (3-42), respectively, with no statistically significant difference. The median and range for the proportions of changed genome was $8 \%$ (3.7-19.1\%), 6.2\% (5.9-11.9\%) and 2.2\% (0.09-10.9\%), respectively ( $P=0.06$ Wilcoxon's rank-sum test). The calculations excluded chromosomes $\mathrm{X}$ and Y. All three entities shared many common copy number variations, particularly those with large-scale variation. However, distinct copy number variations were present in each entity (Figure 6). The detailed copy number variation is listed in Supplementary Table 3.

The major discordant copy number variations for intracystic papillary carcinoma were on chr7. More specifically, one sample (no. 13) had focal loss at 7p15.1-14.1 and p12.1-q11.21, whereas two samples had p arm gain. The same sample (no. 13) had q21.11 gain, whereas three samples q arm loss.

Specific genome variation between intracystic papillary carcinoma and ductal carcinoma in situ. The mean probability of copy number changes for ductal 
a

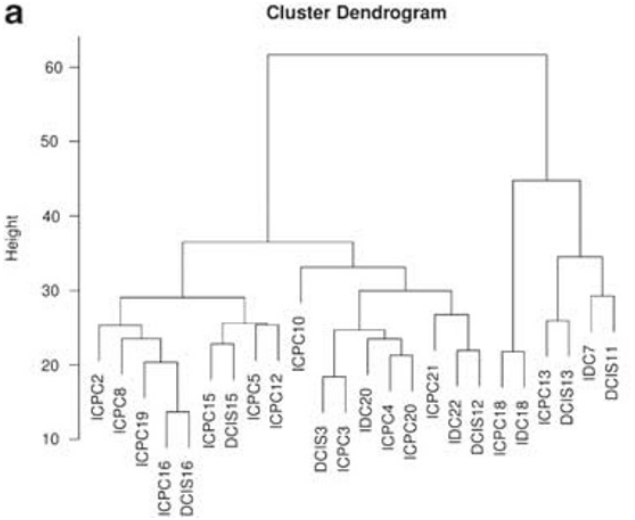

b

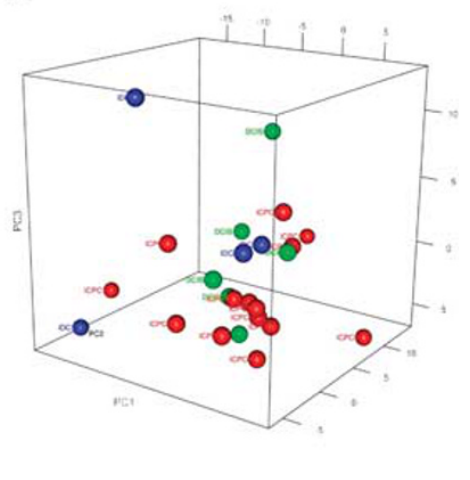

Figure 6 Clustering intracystic papillary carcinoma (ICPC) with concurrent ductal carcinoma in situ (DCIS) and invasive ductal carcinoma (IDC) using (a) cluster dendogram and (b) principal component analysis.

Table 2 Acquired cell adhesion and cell motility genes in DCIS

\begin{tabular}{|c|c|c|c|}
\hline Gene & Status & Location & Function \\
\hline$S P A G 1^{24}$ & Gain & $8 \mathrm{q} 22.2$ & Procell motility \\
\hline$R H P N 1^{25}$ & Gain & $8 \mathrm{q} 24.3$ & $\begin{array}{l}\text { Coordinated assembly of focal } \\
\text { adhesions }\end{array}$ \\
\hline$S C R I B^{26}$ & Gain & $8 \mathrm{q} 24.3$ & Procell migration and cell polarity \\
\hline MIR661 27 & Loss & $11 \mathrm{~g} .25$ & Anticell motility \\
\hline$N T M^{28}$ & Loss & $11 \mathrm{q} .25$ & $\begin{array}{l}\text { GPI-anchored cell adhesion } \\
\text { molecule }\end{array}$ \\
\hline$O P C M L^{28}$ & Loss & $11 \mathrm{q} .25$ & Cell adhesion molecule \\
\hline$I A M 3^{29}$ & Loss & $11 \mathrm{~g} .25$ & Junctional adhesion molecule \\
\hline
\end{tabular}

carcinoma in situ was similar to intracystic papillary carcinoma with the following additional focal changes that was seen in ductal carcinoma in situ compared with intracystic papillary carcinoma: 8q22.2-22.3 gain (from 100114836 to 102268083 , in 3 of 6 cases), 8q24.3 gain (from 142202743 to 145957473 , in 3 of 6 cases), 11q25 loss (from 131565893 to 133557293 , in 3 of 6 cases) and 12q24.31-24.33 loss (from 124302840 to 129818768 , in 2 of 6 cases). Supplementary Table 4 lists all the changes with the involved genes. Interestingly, many of these genes are involved in the cell adhesion and cell motility (Table 2).

There was one strong focal change within 1q gain: 1q21.3-23.1 (8 of 14 cases) (from 153183579 to 155186038 ) in intracystic papillary carcinoma. This focus was not seen in concurrent ductal carcinoma in situ. The genes located in this region are presented in Supplementary Table 5. Two genes are of interest, papillary renal cell carcinoma (PRCC) and neutrotophic tyrosine kinase receptor type 1 (NTRK1).

\section{Discussion}

Classifying intracystic papillary carcinoma under ductal carcinoma in situ or invasive ductal carcinoma is still a matter of debate and the biology and pathogenesis are still unclear. It was proposed that intracystic papillary carcinoma is a form of invasive ductal carcinoma for the following reasons: (1) Lack of the surrounding myoepithelial cell layer at the tumor-stromal interface in intracystic papillary carcinoma, a known feature for invasive ductal carcinoma. $^{3,5}$ However, lack of myoepithelial cell layer is not always indicative of invasion; microglandular adenosis is a good example., ${ }^{46}$ (2) Axillary lymph node or distant metastases in intracystic papillary carcinoma that lacks unequivocal invasion is well known. ${ }^{17}$ However, up to $7 \%$ of ductal carcinoma in situ could develop nodal metastases with no evidence of invasion. ${ }^{18}$ In addition, tumor local invasion may be related to tumor displacement ${ }^{19}$ and lymph node metastasis may be the result of synchronous separate occult invasive ductal carcinoma. ${ }^{20}$ Therefore, local recurrence and/or lymph node metastases do not make intracystic papillary carcinoma an invasive carcinoma. In conclusion and based on the known indolent behavior of this tumor, the WHO task force recommended that intracystic papillary carcinoma be staged and treated like ductal carcinoma in situ. ${ }^{1}$

There have been few studies that have investigated the biology of intracystic papillary carcinoma. Using markers of invasion, Rakha et $a l^{21}$ found that intracystic papillary carcinoma exhibited an expression pattern of invasion-associated markers between ductal carcinoma in situ and invasive ductal carcinoma, concluding that this tumor has unique biological features. Duprez et $a l^{9}$ investigated whether papillary carcinomas would constitute an entity distinct from invasive ductal carcinoma at the genomic level, or whether they would merely constitute a morphological variant of estrogen receptorpositive invasive ductal carcinoma. They included 39 intracystic papillary carcinomas and compared with grade- and estrogen receptor-matched nonconcurrent invasive ductal carcinoma. They suggested that papillary carcinoma belongs to a spectrum of estrogen receptor-positive invasive ductal carcinoma. They also hypothesized that papillary 
carcinoma may merely constitute a final stage of development of a ductal carcinoma in situ within a pre-existing papilloma.

Our study is the first to compare between intracystic papillary carcinoma with concurrent ductal carcinoma in situ and invasive ductal carcinoma on the genomic level. Although intracystic papillary carcinoma clustered with concurrent ductal carcinoma in situ and invasive ductal carcinoma, it had more complex gene aberration than the other two. Duprez et $a l^{9}$ found that papillary carcinomas harbor significantly fewer gene copy number aberration than matched invasive ductal carcinoma. The reason for this discordance may be related to one or many reasons: (1) they included all types of papillary carcinomas, and also intracystic papillary carcinoma type; (2) the studied invasive ductal carcinoma was not concurrent; (3) we studied a relatively small number of cases; and finally, (4) most of our intracystic papillary carcinoma cases were of grade II and invasive ductal carcinoma cases of grade I. They also found that papillary carcinoma (invasive ductal carcinoma and intracystic papillary carcinoma) did not form a separate cluster from matching invasive ductal carcinoma. The pattern of genomic aberration found in papillary carcinoma was similar to that of grade- and estrogen receptor-matched invasive ductal carcinoma. ${ }^{9}$ Our study had similar results, as intracystic papillary carcinoma clustered with invasive ductal carcinoma as well as with concurrent ductal carcinoma in situ with minimal aberration. However, it is worth noting that the number of studied cases is very small, particularly invasive ductal carcinoma, for the results to be definitive.

We found that intracystic papillary carcinoma clustered with two of two invasive ductal carcinoma and four of five ductal carcinoma in situ. This finding would suggest that intracystic papillary carcinoma is closer to invasive ductal carcinoma than to ductal carcinoma in situ. However, the number of studied cases was too small, particularly for invasive ductal carcinoma $(n=2)$ to draw this conclusion. Therefore, we relied on the total number and percentage of genomic changes present in all three entities. The order of genomic change was as follows: intracystic papillary carcinoma, ductal carcinoma in situ and then invasive ductal carcinoma with borderline statistical significance.

There are three major theories about the tumorigenesis and progression of different tumors. The first theory is that the proposed tumors each start with different clone/clones. Thereafter, they acquire mutations and evolve into different tumors independently and in parallel. The second theory is that the normal cell acquires single or set of clones, which produces a type of tumor. Thereafter, this tumor acquires another set of mutations producing a completely different tumor type. The third theory is that the tumors arise from the same ancestral clone. At certain stage, they split into different tumor types and acquire extra changes. ${ }^{22,23}$
In the light of these theories and our findings of variable gene aberrations in different types of intracystic papillary carcinoma, we believe that intracystic papillary carcinoma is genetically not one disease. Intracystic papillary carcinoma with concurrent invasive ductal carcinoma might have different progression pathway and pathogenesis than intracystic papillary carcinoma with concurrent ductal carcinoma in situ (Figure 7). When we compared intracystic papillary carcinoma with ductal carcinoma in situ vs without ductal carcinoma in situ, we found that the latter had gain in small loci 5q35.3, 8q24.3 and 21q13.2. Similarly, when we compared intracystic papillary carcinoma with invasive ductal carcinoma vs without invasive ductal carcinoma, we found that the latter had two changes, 11q22.1-23.3 loss and chr5 gain.

Although intracystic papillary carcinoma with or without ductal carcinoma in situ had similar major chromosomal changes (16p gain, 16q loss, 1q gain and $7 q$ loss), intracystic papillary carcinoma with invasive ductal carcinoma, in addition, had chr5 gain. Therefore, we believe that when intracystic papillary carcinoma develops through acquired gains/losses of major chromosomes, it may start with a set of major changes (16p gain, 16q loss, 1q gain and $7 q$ loss) and then later acquires another major change (chr5 gain) when it progresses into intracystic papillary carcinoma with invasive ductal carcinoma. The other possibility is that intracystic papillary carcinoma without invasive ductal carcinoma started separately and in parallel with intracystic papillary carcinoma with invasive ductal carcinoma.

Interestingly, we found that intracystic papillary carcinoma with invasive ductal carcinoma had 11q22.1-23.3 gain when compared with intracystic papillary carcinoma without invasive ductal carcinoma. In this region, there are multiple MMPS genes. These genes are known to have major role in tumor invasiveness. ${ }^{24}$ These genes might be responsible for giving intracystic papillary carcinoma the capability for invasion and forming invasive ductal carcinoma. However, we could not compare intracystic papillary carcinoma $v s$ invasive ductal carcinoma because of the small number of invasive ductal carcinoma cases that had successful gene array.

For the pathogenesis of pure intracystic papillary carcinoma vs intracystic papillary carcinoma with ductal carcinoma in situ, we believe that they both start with major chromosomal changes (16p gain, $16 q$ loss, $1 q$ gain and $7 q$ loss). They differ in additional minor chromosomal changes (Figure 7).

When intracystic papillary carcinoma was compared with concurrent ductal carcinoma in situ, we found that the former had 1q21.3-23 gain. In this region, there are two genes of interest, $P R C C$ and NTRK1, which are altered in papillary carcinomas of the kidney and thyroid, respectively. ${ }^{25,26}$ Chromosomal rearrangements involving the NTRK1 gene are found in approximately $10 \%$ of thyroid papillary carcinoma. They result from the fusion of 


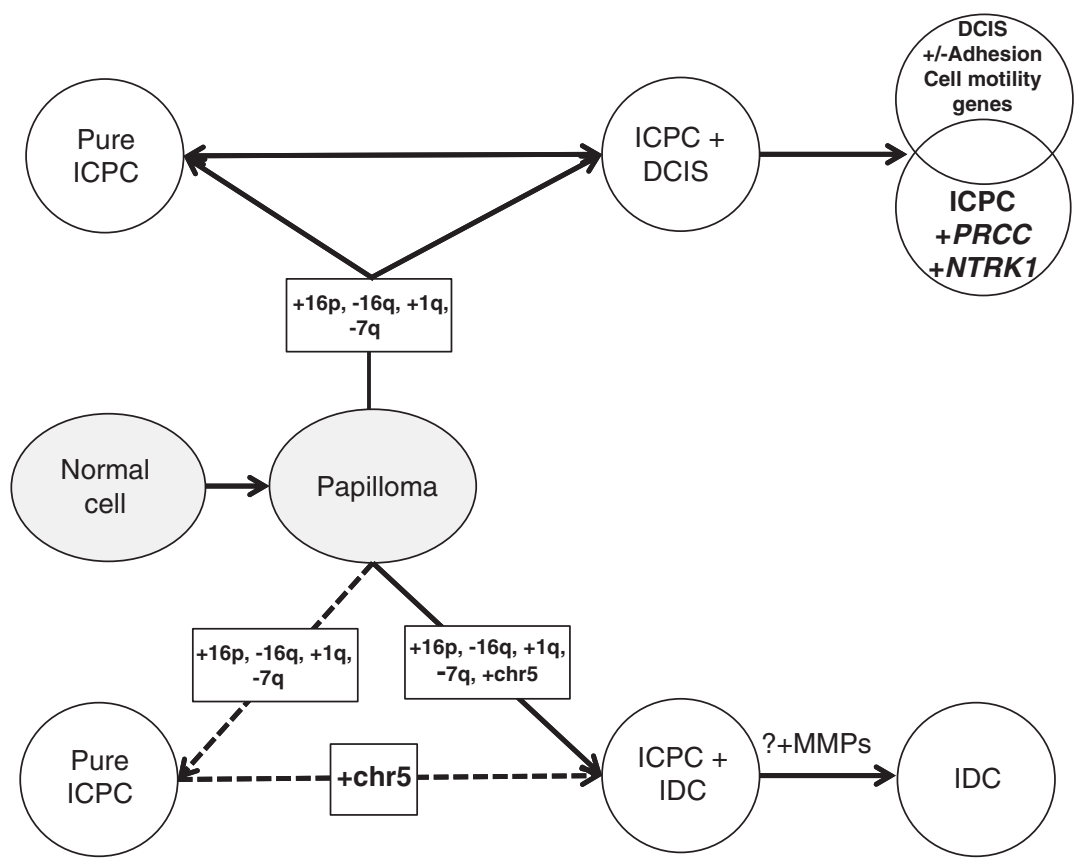

Figure 7 Proposed pathogenesis of intracystic papillary carcinoma (ICPC) with relation to ductal carcinoma in situ (DCIS) and invasive ductal carcinoma (IDC).

the tyrosine kinase domain of TRK to $5^{\prime}$-terminal sequence of the tropomyosin (TMP3), or TPR genes also located on chromosome 1q, or of the TFG gene on chromosome $3 .^{26}$ PRCC is one of several genes that are involved in the renal cell carcinoma type that is associated with Xp11.2 translocations/TFE3 gene fusions. ${ }^{25}$ One of the interesting points of papillary carcinoma of the kidney and the thyroid is that these tumors have more indolent behavior compared with their organ-related counterparts. ${ }^{25,26}$ It is known that intracystic papillary carcinoma has an indolent behavior as well. ${ }^{1}$ Therefore, this frequent change in intracystic papillary carcinoma but not ductal carcinoma in situ could explain the papillary morphology and its indolent clinical behavior. Duprez et $a l^{9}$ did not find a determinant of the papillary phenotype of papillary carcinoma of the breast (invasive ductal carcinoma and intracystic papillary carcinoma). They suggested that this determination could be related to other changes including genetic aberrations other than gene copy number aberrations (ie copy number silent loss of heterozygosity events, somatic mutations or fusion genes), epigenetic changes or distinctive tumor-microenvironment interactions. Although we found amplification of these two genes (through 1q21.3-23 gain), rather than translocation (like in $P R C C$ ) or rearrangement (like NTRK1), this finding could be useful for future investigation. Although there have been no studies to correlate between PRCC and breast cancer, Dolle et $a l^{27}$ have demonstrated an autocrine NGF/NTRK1 loop involvement in breast cancer progression.

When ductal carcinoma in situ is compared with concurrent intracystic papillary carcinoma, we found that the former had a set of copy number changes including 8q22.2-22.3 gain, 8q24.3 gain, 11q25 loss and 12q24.31-24.33 loss. We found a gene set of interest that are all involved in cell adhesion and cell motility. Table 2 shows the genes names and their description. These genes differ in their function, pro- or antiadhesion. ${ }^{28-33}$ Ductal carcinoma in situ has variable expression of these genes (gain or loss). Therefore, we believe that the function of cell-to-cell adhesion is significantly altered. We suggest that the motility and adhesion of cell molecular changes permit the neoplastic cells to migrate from intracystic papillary carcinoma and colonize in the surrounding ducts, but do not give it the required mechanism for stromal invasion.

We conclude that intracystic papillary carcinoma is genetically closer to ductal carcinoma in situ than to invasive ductal carcinoma, which may explain the indolent behavior of this tumor. Intracystic papillary carcinoma shares major copy number variation with minimal but significant genetic variation that may have a critical role in the pathogenesis and biology of this tumor. Further studies with larger number of cases are needed to verify our results.

\section{Acknowledgements}

We thank Ms Ashley Vigars for her secretarial and clerical assistance.

\section{Disclosure/conflict of interest}

The authors declare no conflict of interest. 


\section{References}

1 Lakhani SR, Schnitt SJ, O’Malley F, et al. Lobular neoplasia, In: Lakhani SR, Ellis IO, Schnitt SJ, Tan PH, van de Vijver MJ (eds). World Health Organization Classification of Tumors: Pathology and Genetics of Tumors of the Breast and Female Genital Organs. IARC Press: Lyon, France; 2012, pp 106-107.

2 Calderaro J, Espie M, Duclos J, et al. Breast intracystic papillary carcinoma: an update. Breast J 2009;15: 639-644.

3 Collins LC, Carlo VP, Hwang $\mathrm{H}$, et al. Intracystic papillary carcinomas of the breast: a reevaluation using a panel of myoepithelial cell markers. Am J Surg Pathol 2006;30:1002-1007.

4 Esposito NN, Dabbs DJ, Bhargava R. Are encapsulated papillary carcinomas of the breast in situ or invasive? A basement membrane study of 27 cases. Am J Clin Pathol 2009;131:228-242.

5 Hill CB, Yeh IT. Myoepithelial cell staining patterns of papillary breast lesions: from intraductal papillomas to invasive papillary carcinomas. Am J Clin Pathol 2005;123:36-44.

6 Di Cristofano C, Mrad K, Zavaglia K, et al. Papillary lesions of the breast: a molecular progression? Breast Cancer Res Treat 2005;90:71-76.

7 Lininger RA, Park WS, Man YG, et al. LOH at 16p13 is a novel chromosomal alteration detected in benign and malignant microdissected papillary neoplasms of the breast. Hum Pathol 1998;29:1113-1118.

8 Tsuda H, Fukutomi T, Hirohashi S. Pattern of gene alterations in intraductal breast neoplasms associated with histological type and grade. Clin Cancer Res 1995;1:261-267.

9 Duprez R, Wilkerson PM, Lacroix-Triki M, et al. Immunophenotypic and genomic characterization of papillary carcinomas of the breast. J Pathol 2012;226:427-441.

10 Elston CW, Ellis IO. Pathological prognostic factors in breast cancer. I. The value of histological grade in breast cancer: experience from a large study with longterm follow-up. Histopathology 1991;19:403-410.

11 Holland R, Peterse JL, Millis RR, et al. Ductal carcinoma in situ: a proposal for a new classification. Semin Diagn Pathol 1994;11:167-180.

12 Nowak NJ, Miecznikowski J, Moore SR, et al. Challenges in array comparative genomic hybridization for the analysis of cancer samples. Genet Med 2007;9:585-595.

13 Olshen AB, Venkatraman ES, Lucito R, et al. Circular binary segmentation for the analysis of arraybased DNA copy number data. Biostatistics 2004;5: $557-572$.

14 Van de Wiel MA, Kim KI, Vosse SJ, et al. CGHcall: calling aberrations for array CGH tumor profiles. Bioinformatics 2007;23:892-894.

15 Van de Wiel MA, Smeets SJ, Brakenhoff RH, et al. CGHMultiArray: exact P-values for multi-array comparative genomic hybridization data. Bioinformatics 2005;21:3193-3194.

16 Collins LC, Schnitt SJ. Papillary lesions of the breast: selected diagnostic and management issues. Histopathology 2008;52:20-29.
17 Solorzano CC, Middleton LP, Hunt KK, et al. Treatment and outcome of patients with intracystic papillary carcinoma of the breast. Am J Surg 2002;184:364-368.

18 Katz A, Gage I, Evans S, et al. Sentinel lymph node positivity of patients with ductal carcinoma in situ or microinvasive breast cancer. Am J Surg 2006;191:761-766.

19 Nagi C, Bleiweiss I, Jaffer S. Epithelial displacement in breast lesions: a papillary phenomenon. Arch Pathol Lab Med 2005;129:1465-1469.

20 Dzodic R, Stanojevic B, Saenko V, et al. Intraductal papilloma of ectopic breast tissue in axillary lymph node of a patient with a previous intraductal papilloma of ipsilateral breast: a case report and review of the literature. Diagn Pathol 2010;5:17.

21 Rakha EA, Tun M, Junainah E, et al. Encapsulated papillary carcinoma of the breast: a study of invasion associated markers. J Clin Pathol 2012;65:710-714.

22 Walter MJ, Shen D, Ding L, et al. Clonal architecture of secondary acute myeloid leukemia. N Engl J Med 2012;22:1090-1098.

23 Yates LR, Campbell PJ. Evolution of the cancer genome. Nat Rev Genet 2012;13:795-806.

24 Egeblad M, Werb Z. New functions for the matrix metalloproteinases in cancer progression. Nat Rev Cancer 2002;2:161-174.

25 Argani P, Ladanyi M. renal cell carcinoma associated with Xp11.2 translocation/TFE3 gene fusions, In: Eble JN, Sauster G, Epstein JI, Sesterhenn IA (eds) World Health Organization Classification of Tumors: Pathology and Genetics of Tumors of the Urinary Tract System and Male Genital Organs. IARC Press: Lyon, France; 2004, pp 37-38.

26 LiVolsi VA, Albores-Saavedra J, Asa SL, et al. Papillary carcinoma, In: DeLellis A, Lloyd RV, Heitz PU, Eng C (eds) World Health Organization Classification of Tumors: Pathology and Genetics of Tumors of Endocrine Organs. IARC Press: Lyon, France; 2004, pp 57-66.

27 Dolle R, Adriaenssens E, El Yazidi-Belkloura I, et al. Nerve growth factor receptors and signaling in breast cancer. Curr Cancer Drug Targets 2004;4:463-470.

28 Neesse A, Gangeswaran R, Luettges J, et al. Spermassociated antigen 1 is expressed early in pancreatic tumorigenesis and promotes motility of cancer cells. Oncogene 2007;26:1533-1545.

29 Ridley AJ, Hall A. The small GTP-binding protein rho regulates the assembly of focal adhesions and actin stress fibers in response to growth factors. Cell 1992;70:389-399.

30 Nola S, Sebbagh M, Marchetto S, et al. Scrib regulates PAK activity during the cell migration process. Hum Mol Genet 2008;17:3552-3565.

31 Reddy SDN, Pakala SB, Ohshiro K, et al. MicroRNA661, a c/EBP-alpha target, inhibits metastatic tumor antigen 1 and regulates its functions. Cancer Res 2009;69:5639-5642.

32 Sellar GC, Watt KP, Rabiasz GJ, et al. OPCML at 11q25 is epigenetically inactivated and has tumor-suppressor function in epithelial ovarian cancer. Nature Genet 2003;34:337-343.

33 Arrate MP, Rodriguez JM, Tran TT, et al. Cloning of human junctional adhesion molecule 3 (JAM3) and its identification as the JAM2 counter-receptor. J Biol Chem 2001;276:45826-45832. 